\title{
High-field diffusion tensor imaging characterization of cerebral white matter injury in lipopolysaccharide-exposed fetal sheep
}

\author{
Yohan van de Looij ${ }^{1}, 2$, Gregory A. Lodygensky', Justin Dean ${ }^{3}$, François Lazeyras ${ }^{4}$, Henrik Hagberg ${ }^{5}$, Ingemar Kjellmer ${ }^{6}$, \\ Carina Mallard ${ }^{3}$, Petra S. Hüppi ${ }^{1}$ and Stéphane V. Sizonenko ${ }^{1}$
}

BACKGROUND: In gyrencephalic species such as sheep, precise anatomical and microstructural characterization of the consequences of fetal inflammation remains scarce. The goal of this study was to characterize changes in white matter (WM) structure using advanced magnetic resonance imaging (MRI) following lipopolysaccharide (LPS) exposure in the pretermequivalent fetal sheep.

METHODS: Preterm (0.7 gestation) fetal sheep received vehicle (Sham group) or LPS (LPS group), and fetal brains were collected $10 \mathrm{~d}$ later for subsequent ex vivo MRI. T,-weighted (T,W), $\mathrm{T}_{2}$-weighted $\left(\mathrm{T}_{2} \mathrm{~W}\right)$, and diffusion tensor imaging (DTI) data were collected.

RESULTS: Fetuses exposed to LPS exhibited reductions in WM volume and corpus callosum thickness at $10 \mathrm{~d}$ recovery. Characteristic patterns of diffuse and focal WM lesions (necrosis or cysts) could be identified by various $T_{1}, T_{2}$, and DTI signal changes.

CONCLUSION: Fetal LPS exposure induces a pattern of injury characterized by diffuse and focal WM injury that closely reproduces that observed clinically in preterm infants. This work provides anatomical and microstructural MRI assessment, as well as histopathological correlates, of the consequences of LPS exposure in an animal model with a WM structure similar to that of the human brain. This work will help to further our understanding of MRI changes in preterm infants.

P eriventricular leukomalacia (PVL) is classically described as focal cystic lesions in the white matter (WM) (1) and is seen in less than $10 \%$ of preterm infants. More commonly, diffuse WM abnormalities are present in early preterm infants, with up to $79 \%$ of infants showing magnetic resonance imaging (MRI) abnormalities in WM (2). Through a number of MRI studies it has been shown that classical PVL does not represent the only features of preterm brain injury (3). A more diffuse component involving WM globally and also gray matter (GM) with altered development has also been described. A new concept of encephalopathy of prematurity with focal and/or diffuse WM injury, but also with cerebral development alteration including GM, is now developing (3). Focal injury can consist of necrosis in the deep WM, with loss of all cellular elements, that evolves to cystic lesions (3). Other focal lesions often observed are punctate lesions, which correspond to focal rarefaction of myelin, loss of fibers, or lacunar infarctions (4), and associated with altered microstructure and myelination (5). The diffuse abnormalities, defined as diffuse excessive high-signal intensity on MRI (6), have been associated with the presence of astrogliosis and microgliosis, and initially with a decrease in premyelinating oligodendrocytes (3). Nevertheless, it is still unclear whether diffuse excessive highsignal intensity results from a destructive disease or altered WM development.

There is now strong epidemiological $(7,8)$ and experimental $(9,10)$ evidence for a role of maternal/fetal inflammation in preterm brain injury. For example, in preterm fetal sheep, systemic administration of lipopolysaccharide (LPS) causes cerebral inflammation and diffuse WM damage consisting of a loss of immature oligodendrocytes and altered myelination, as well as more focal damage such as cystic and gliotic lesions, in the periventricular WM (PVWM) and subcortical WM $(11,12)$. This pattern of damage is remarkably similar to the diffuse and focal WM lesions observed clinically in preterm brain injury (11).

MRI is a powerful tool to noninvasively assess brain development and injury. A combination of $\mathrm{T}_{1}$-weighted $\left(\mathrm{T}_{1} \mathrm{~W}\right)$ and $\mathrm{T}_{2}$-weighted ( $\left.\mathrm{T}_{2} \mathrm{~W}\right)$ MRI can be used to detect and differentiate the cysts, punctate lesions, and diffuse excessive high-signal intensity observed in preterm infants. Measurement of specific cerebral tissue volumes can be achieved by segmentation of the MRI into tissue types, on the basis of the contrast of the MRI (13). Several studies have assessed the developmental consequences of WM injury in the preterm infant on brain tissue volumes at term $(14,15)$ and later in life $(16)$, showing loss of WM and cortical volumes. Diffusion tensor imaging (DTI) is an advanced MRI technique widely used to explore brain microstructure development and injury (17-21). The combination of fractional anisotropy (FA) and diffusivity values (axial and

'Department of Pediatrics, University of Geneva, Geneva, Switzerland; ${ }^{2}$ Laboratory of Functional and Metabolic Imaging, Ecole Polytechnique Fédérale de Lausanne, Lausanne, Switzerland; ${ }^{3}$ Department of Neuroscience and Physiology, University of Gothenburg, Gothenburg, Sweden; ${ }^{4}$ Department of Radiology, University of Geneva, Geneva, Switzerland; ${ }^{5}$ Department of Obstetrics and Gynecology, University of Gothenburg, Gothenburg, Sweden; ${ }^{6}$ Department of Pediatics, University of Gothenburg, Gothenburg, Sweden. Correspondence: Yohan van de Looij (yohan.vandelooij@epfl.ch) 
radial) derived from DTI gives a very accurate assessment of tissue organization, making DTI a powerful tool to assess WM abnormalities following LPS (18) or ischemia (19). The aim of this study was to provide MRI delineation of the WM changes in the brain following exposure to LPS in the 0.7-gestation fetal sheep (corresponding to human preterm at 28-32 wk of gestation). Multimodal MRI techniques including DTI, $\mathrm{T}_{1} \mathrm{~W}$, and $\mathrm{T}_{2} \mathrm{~W}$ were used in combination with quantitative segmentation image analysis and histopathology to define the characteristic signatures of WM cysts, punctate lesions, and diffuse excessive high-signal intensity.

\section{RESULTS}

\section{Volumetric Changes After LPS Challenge}

High-resolution $\mathrm{T}_{1}$ and $\mathrm{T}_{2}$ data sets together with a k-nearestneighbor classification algorithm optimized for immature brains allowed successful segmentation of the cohort (Figure 1). Reproducibility tests were not performed and after verification of the results, this technique did not require fine editing. Fetal sheep brains have a similar contrast and brain tissue composition to those of human newborns, allowing us to use a well-established human segmentation technique $(13,22)$. It should be noted that before magnetic resonance (MR) experiments the brains were bathed with paraformaldehyde (PFA) for preservation, whereas during MR experiments the brains were bathed with fomblin (inert oil without any MR signal). Nevertheless, some traces of PFA remained present in the sample, resulting in $\mathrm{T}_{2} \mathrm{~W}$ images showing hyperintensities in the ventricles and on the outer surface of the brain. As such, signal intensity from PFA was classified
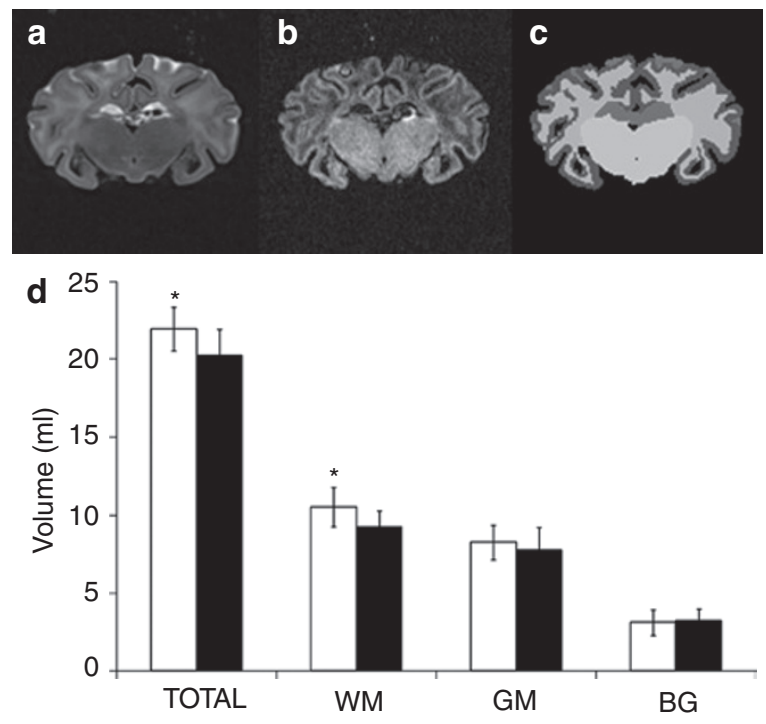

Figure 1. Brain volume segmentation. (a) Coronal $\mathrm{T}_{2} \mathrm{~W}$ image, (b) $\mathrm{T}_{1} \mathrm{~W}$ image, and (c) corresponding segmented image, with the following color code: dark gray is gray matter (GM), light gray is white matter (WM), white is the basal ganglia (BG), and gray is nonliving liquid (i.e., formalin situated in the ventricles and outside of the brain). (d) Histogram of the volumetric measurements of the total brain volume (TOTAL), WM, GM, and BG expressed in $\mathrm{ml}$ as mean \pm SD for the Sham (white) and LPS (black) groups $\left({ }^{*} P<0.05\right)$. LPS, lipopolysaccharide; $T_{1} W, T_{1}$-weighted; $T_{2} W, T_{2}$-weighted. as nonliving liquids. Due to the presence of PFA, volumetric estimation of the ventricles on the basis of signal intensity was not performed. LPS exposure was associated with a significant reduction in total brain volume (i.e., $\mathrm{WM}+\mathrm{GM}+$ basal ganglia) (Sham group: $21.9 \pm 1.4 \mathrm{ml}$ vs. LPS group: $20.3 \pm 1.7 \mathrm{ml}$, $P=0.02$ ) and WM volume (Sham group: $10.5 \pm 1.2 \mathrm{ml}$ vs. LPS group: $9.2 \pm 1.0 \mathrm{ml}, P=0.02$ ). There were no differences in GM and basal ganglia volumes between the two groups. There was also a significant reduction in the thickness of the corpus callosum (CC) following LPS exposure (Sham group: $3.2 \pm$ $0.9 \mathrm{~mm}$, LPS group: $2.1 \pm 0.6 \mathrm{~mm}, P=0.004)$.

\section{Microstructure Assessment After LPS Challenge Using DTI}

DTI in CC and PVWM. For the principal WM structures analyzed-CC and PVWM on the anterior, median, and posterior levels (Figures 2 and 3)-FA values were significantly lower in the LPS group than in the Sham group. The mean FA values (i.e., mean values over the three different image planes) in the CC were $0.78 \pm 0.04$ and $0.64 \pm 0.06$ for the Sham group and LPS group, respectively, and the mean FA values in the PVWM were $0.66 \pm 0.07$ and $0.49 \pm 0.09$ for the Sham group and LPS group, respectively. The decrease of FA was predominantly due to a significant increase of radial diffusivity $\left(D_{\perp}\right)$ at the anterior level of the CC only, whereas $\mathrm{D}_{\perp}$ tended to be higher in the LPS group than in the Sham group for all other brain levels in the CC and PVWM. Over the three levels, $\mathrm{D}_{\perp}$ in the CC was $1.27 \times 10^{-4} \pm 0.49 \times 10^{-4}$ $\mathrm{mm}^{2} / \mathrm{s}$ in the Sham group and $1.76 \times 10^{-4} \pm 0.60 \times 10^{-4} \mathrm{~mm}^{2} / \mathrm{s}$ in the LPS group, and $D_{\perp}$ in the PVWM was $1.58 \times 10^{-4} \pm 0.61$ $\times 10^{-4} \mathrm{~mm}^{2} / \mathrm{s}$ in the Sham group and $2.27 \times 10^{-4} \pm 0.73 \times 10^{-4}$ $\mathrm{mm}^{2} / \mathrm{s}$ in the LPS group. Mean diffusivity (MD) and axial diffusivity $\left(\mathrm{D}_{\|}\right)$were not different between the two groups. There were no significant differences in FA values between the LPS and Sham groups in the cortex or internal or external capsules (data not shown).

Correlations with global score of WM injury. Next, we calculated global WM abnormality scores in the CC and PVWM for each animal, and correlated with changes in DTI data. In the $\mathrm{CC}$, there was a significant correlation between clinical score and FA $(r=-0.67, P=0.049), \mathrm{D}_{\perp}(r=0.75, P=0.021), \mathrm{D}_{\mu}(r=$ $0.67, P=0.047)$, and $\mathrm{MD}(r=0.72, P=0.029)$ (Figure 4). There were no significant correlations between injury scores and DTI data in the PWVM (data not shown).

DTI assessment of MRI signal abnormalities. Focal lesions (with the two opposite patterns of $\mathrm{T}_{1}$ and $\mathrm{T}_{2}$ signal abnormalities) in the LPS group were always observed in the fronto-parietal PVWM, whereas diffuse WM signal abnormalities were observed at different sites in the subcortical WM throughout the brain. Note that subcortical WM relates to WM adjacent to the cortical rim and extends toward the PVWM.

Typical diffuse WM lesions localized in the intragyral WM were identified on conventional imaging characterized by hypointensity on $\mathrm{T}_{1} \mathrm{~W}$ images and hyperintensity on $\mathrm{T}_{2} \mathrm{~W}$ images in seven of nine brains exposed to LPS. These diffuse WM changes in signal intensity were disseminated in the subcortical WM throughout the brain. 


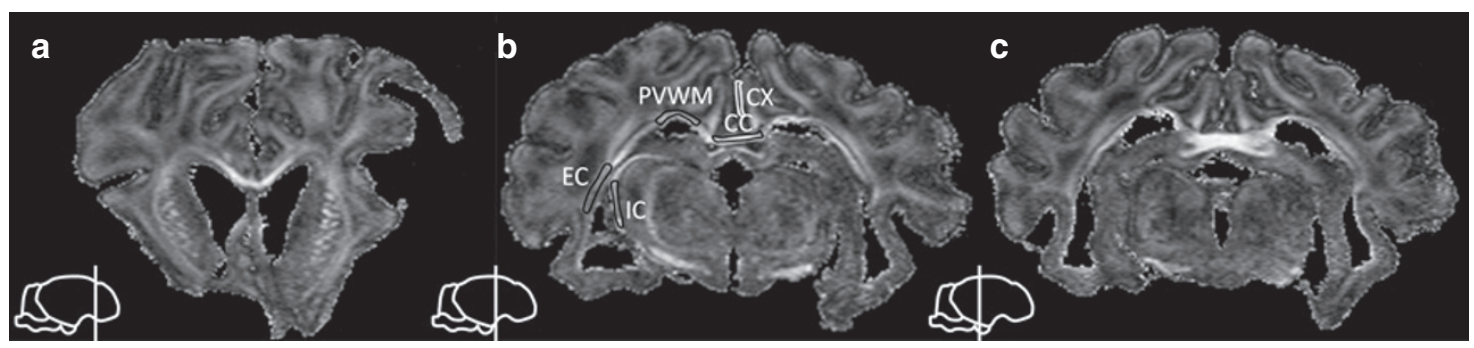

Figure 2. Fractional anisotropy maps of the three different image planes analyzed: (a) anterior, (b) median, and (c) posterior. Regions of interest analyzed overlaid on the median map: the corpus callosum (CC), the periventricular white matter (PVWM), the internal capsule (IC), the external capsule (EC), and the cortex (CX).
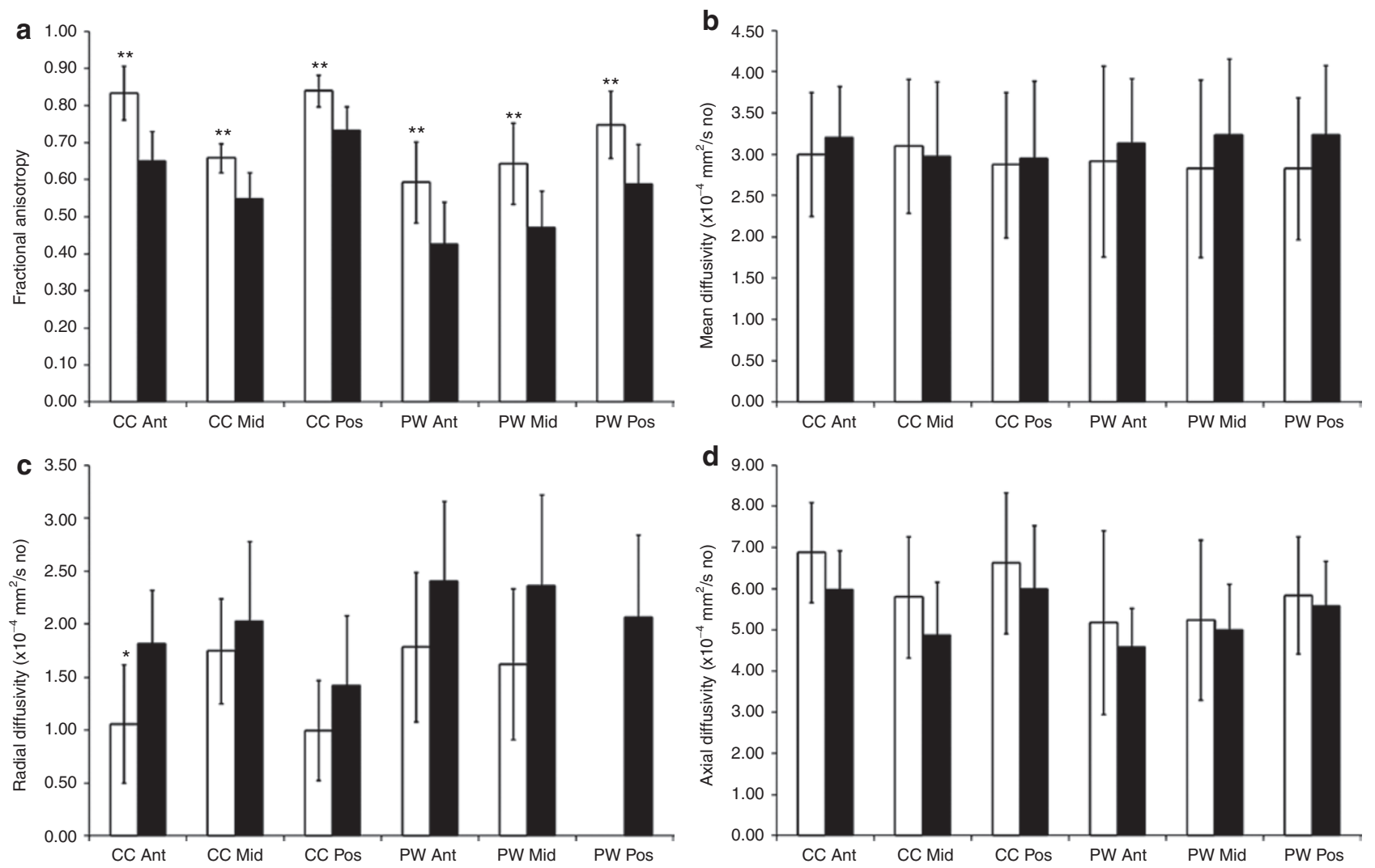

Figure 3. DTI-derived parameters in the corpus callosum and periventricular white matter. (a) Mean fractional anisotropy and diffusivity values, (b) mean, (c) radial, and (d) axial \pm SD measured in the corpus callosum (CC) and periventricular white matter (PVWM) at anterior (Ant), median (Med), and posterior (Pos) levels of the brain for the Sham group (white) and the LPS group (black). ${ }^{*} P<0.05$; ${ }^{* * P}<0.01$. Note the significant reduction of anisotropy in the LPS group at the three different image planes analyzed. LPS, lipopolysaccharide.

When compared with the Sham group, FA in diffuse WM lesions was significantly reduced, predominantly due to an increase in $\mathrm{D}_{\perp}$ (Figure 5). Corresponding areas of altered $\mathrm{T}_{1}$ and $\mathrm{T}_{2}$ signal demonstrated marked alteration in acid fuchsin/thionin $(\mathrm{AF} / \mathrm{T})$ staining in histological sections with reduced cellularity. No changes in glial fibrillary acidic protein astrocytes were identified, but evidence of reduced antiphos-neurofilament and amyloid precursor protein (APP) staining was observed in these areas.

Focal lesions were observed in the fronto-parietal PVWM (Figure 6), characterized by well-defined hyperintensity on $\mathrm{T}_{1} \mathrm{~W}$ images and hypointensity on $\mathrm{T}_{2} \mathrm{~W}$ images. These lesions were found in six of nine of the LPS-exposed animals, and were always located either bilaterally or unilaterally in the fronto-parietal PVWM. The volume range of these focal lesions was $3.8 \times 10^{-3}$ to $35 \times 10^{-3} \mathrm{~mm}^{3}$ (mean $=11.8 \times$ $10^{-3} \mathrm{~mm}^{3}$ ). The fibers in these regions were disorganized depicted by a moderately altered pattern of the eigenvectors (Figure 6-necrotic lesion) along with high $\mathrm{D}_{\perp}$ and low FA (Figure 7). In histological sections, areas with lesions identified as "necrotic" by MRI demonstrated a cell necrosis pattern seen on $\mathrm{AF} / \mathrm{T}$ involving cellular elements with neuronal APP accumulation, antiphos-neurofilaments, and glial fibrillary acidic protein loss (Figure 6). Within the lesions, microglia cells with a macrophage appearance were present (Figure 6). 


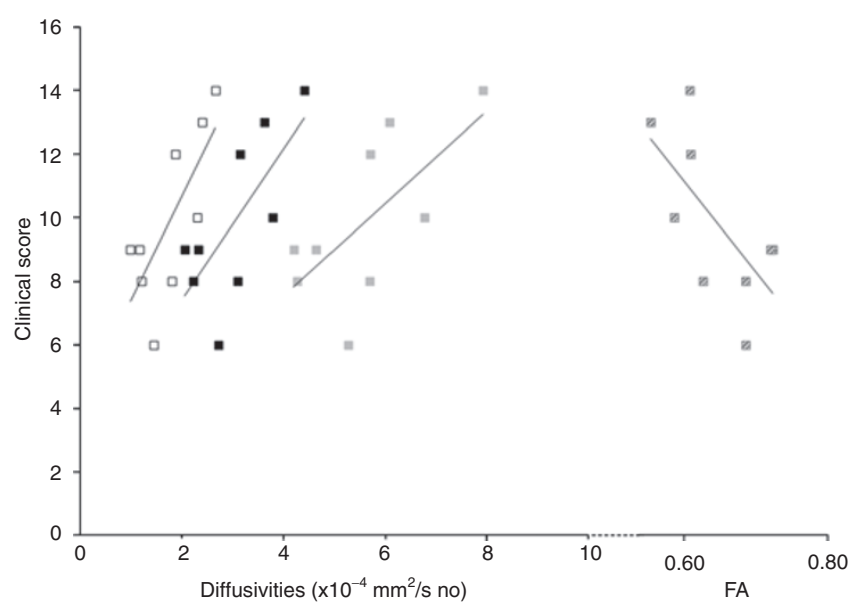

Figure 4. Scatter plots of the clinical score as a function of the diffusion tensor imaging-derived parameters measured in the corpus callosum: radial diffusivity (white box), mean diffusivity (black box), axial diffusivity (gray box), and fractional anisotropy (hatched box). A significant correlation was found between clinical score and radial diffusivity $(r=0.75, P=$ $0.021)$, mean diffusivity $(r=0.72, P=0.029)$, and axial diffusivity $(r=0.67$, $P=0.047)$, as well as fractional anisotropy $(r=-0.67, P=0.049)$.

In addition, typical cystic lesions of PVL were detected on conventional imaging for only three of nine LPS animals with very strong hyposignal on $\mathrm{T}_{1} \mathrm{~W}$ images and hypersignal on $\mathrm{T}_{2} \mathrm{~W}$ images, very high $\mathrm{MD}$ and $\mathrm{D}_{\perp}$, resulting in very low $\mathrm{FA}$ (comparable to the FA value of cerebrospinal fluid or free water), and a large disruption of WM fibers at the lesion site depicted by the absence of diffusion eigenvectors (Figure 6-cystic lesion). Similarly to necrotic lesions, cysts were always situated either bilaterally or unilaterally in the fronto-parietal PVWM with a volume range of $1.9 \times 10^{-3}$ to $13.7 \times 10^{-3} \mathrm{~mm}^{3}$ (mean $=$ $6.5 \times 10^{-3} \mathrm{~mm}^{3}$ ). In both the necrotic and cystic lesions, FA was significantly reduced and $\mathrm{D}_{\perp}$ significantly increased as compared with Sham values (Figure 7). Only for the cystic lesions, the MD was also significantly and drastically increased as compared with the Sham (Figure 7).

\section{DISCUSSION}

In this study, using advanced MRI techniques and analysis approaches, we have shown that exposure to systemic LPS induces both diffuse and focal tissue damage in the preterm fetal sheep brain as well as disruption of normal cerebral WM development. Both gross anatomical and microstructural changes were found. Total cerebral and WM volumes were reduced in the LPS group, providing evidence for an impaired development of the brain following fetal LPS exposure. Similarly, WM volume loss associated with brain weight decrease (11) observed in this model was also found $2 \mathrm{wk}$ following global ischemia on preterm fetal sheep (19). These changes are comparable with what is observed in preterm human infants; WM injury can be related to tissue atrophy as result of the injury itself and/or to an altered brain growth and development $(14,15,23,24)$. However, we were unable to determine which of these mechanisms were predominantly responsible for the changes observed in our model. In addition
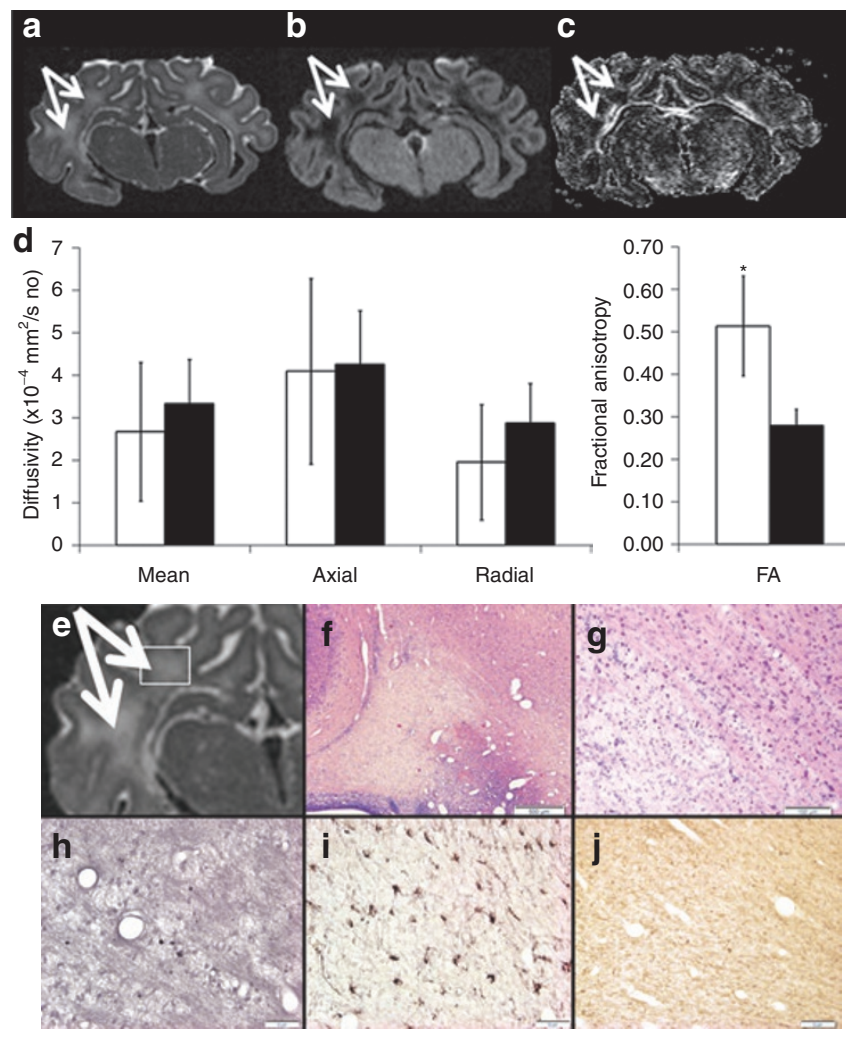

Figure 5. Diffuse white matter lesions. (a) Coronal $T_{1} W$ and (b) coronal $T_{2} W$ images, as well as (c) corresponding fractional anisotropy map of a typical sheep brain exposed to lipopolysaccharide. Arrows indicate altered signal in the white matter for the lipopolysaccharide-infused sheep with hyposignal $\mathrm{T}_{1}$, hypersignal $\mathrm{T}_{2^{\prime}}$ and reduced fractional anisotropy. (d) Corresponding diffusivity and anisotropy values (mean \pm SD) in the lesion (black) as well as in the Sham counterpart (white): a significant reduction of anisotropy was observed in the altered signal $(* P<0.05)$. Histological images of the corresponding area of hyposignal $\mathrm{T}_{1}$ zoomed in (e) (white arrows) are shown in the lower panel: (f) acid fuchsin/thionin, scale bar $=500 \mu \mathrm{m},(\mathbf{g})$ acid fuchsin/ thionin , scale bar $=100 \mu \mathrm{m}$, (h) amyloid precursor protein, scale bar $=50$ $\mu \mathrm{m}$, (i) glial fibrillary acidic protein, scale bar $=50 \mu \mathrm{m}$, and (j) neurofilaments, scale bar $=50 \mu \mathrm{m}$. Sections stained for gross morphological changes by (f, g) acid fuchin/thionin demonstrate regions of diffuse loss of tissue components. These regions contain (h) a few amyloid precursor protein-positive cells, (i) no major change in astrocytes, and (j) a loss of neurofilament staining. The scale bar is overlaid on each histological picture.

to the global decrease in WM volume, we found a significant reduction in the thickness of the CC at $10 \mathrm{~d}$ after LPS exposure. Preterm infants also exhibit altered growth of the CC (25), which is associated with neuromotor delay at $2 \mathrm{y}$ of age (26). Further, reduced thickness of the CC measured by MRI at term-equivalent age in infants born prematurely was predictive of later neurocognitive deficits (27) and was shown to persist into adolescence (28).

At term, several imaging studies have reported reduction in GM volume associated with WM injury $(15,29)$ and cognitive deficits observed in these infants $(27,30)$. Cortical volume loss and reduced expression of neuronal subpopulation was shown in the same animal model (11). In this study, we observed a trend for reduced MRI-derived GM volumes in LPS-treated fetuses. The cortex of the fetal sheep at this age is a very thin 

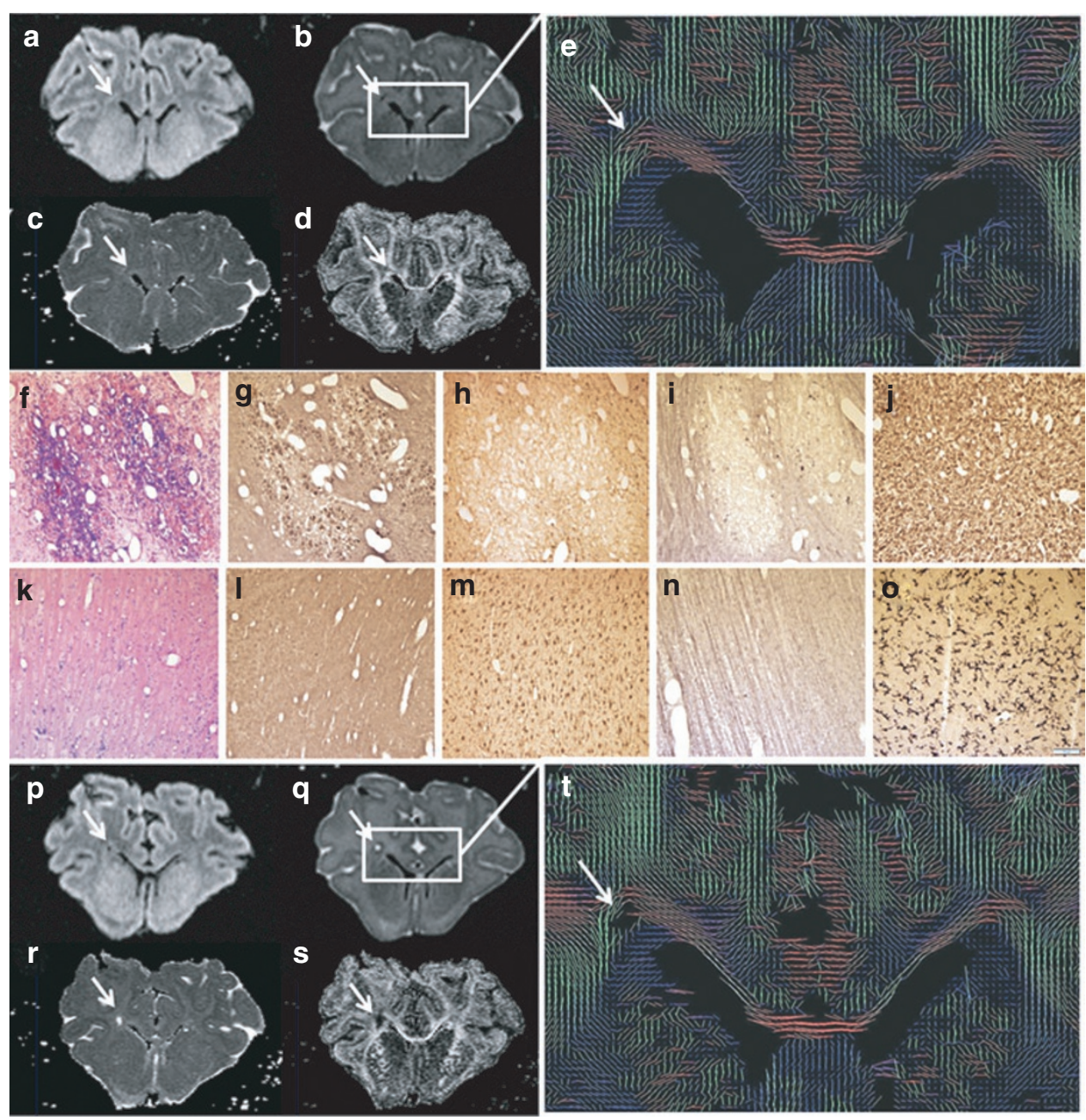

Figure 6. Focal white matter lesions. Upper panel: (a) T,W, (b) T,W images, (c) mean diffusivity, and (d) fractional anisotropy map, as well as (e) directionencoded color map of the eigenvectors zoomed on the lesions of a typical lipopolysaccharide-exposed sheep brain showing necrotic lesion: hypersignal $\mathrm{T}_{1}$, hyposignal $\mathrm{T}_{2}$, low mean diffusivity, low fractional anisotropy (close to the value in gray matter), and moderate disruption of the fibers at the lesion site. Middle panel: histological images of the corresponding areas identified as "necrotic" by magnetic resonance images (a-e: arrows): (f) acid fuchsin/thionin, (g) amyloid precursor protein, (h) glial fibrillary acidic protein, (i) antiphos-neurofilament, and (j) ionized calcium binding adaptor for lipopolysaccharideexposed sheep brain as well as (k) acid fuchsin/thionin, (I) amyloid precursor protein, (m) glial fibrillary acidic protein, (n) antiphos-neurofilament, and (o) ionized calcium binding adaptor Sham counterpart (scale bar $=100 \mu \mathrm{m}$ and is the same for all images and is overlaid on o). Histology demonstrated clear loss of $(\mathbf{h}, \mathbf{m})$ astrocytes and $(\mathbf{i}, \mathbf{n})$ neurofilament, whereas there was an accumulation of $(\mathbf{g}, \mathbf{l})$ amyloid precursor protein-positive cells and an activated (j, o) microglia. Lower panel: (p) T, W, (q) T $T_{2}$ images, (r) mean diffusivity, and (s) fractional anisotropy map, as well as (t) direction-encoded color map of the eigenvectors zoomed on the lesions of a typical lipopolysaccharide-exposed sheep brain showing cystic lesion: strong hyposignal $\mathrm{T}_{1}$ and hypersignal $\mathrm{T}_{2^{\prime}}$ very high mean diffusivity, low fractional anisotropy (close to the value in free water), and no fiber at the lesion site. $T_{1} W_{1} T_{1}$-weighted; $T_{2} W_{1} T_{2}$-weighted.

structure, and partial volume effects of the adjacent tissue in MR images cannot be excluded. As such, accurate assessment of GM volume remains difficult and may contribute to the absence of significant difference between the cortical volumes of the two groups. In addition, the time between the LPS challenge and GM volume measurements (10 d) was relatively short, and therefore, global developmental alterations of GM volume, such as the one depicted by MRI, might not be present yet. With a longer time interval between LPS injury and assessment, one could expect also a more widespread alteration in GM development with reduced volumes. As shown in several studies on human (5) and sheep (19) brains, the pattern of brain lesions depicted by MRI is different as a function of the time point of MR assessment. High-resolution DTI has allowed robust quantification of the DTI-derived parameters $\left(\mathrm{MD}, \mathrm{D}_{\mu}, \mathrm{D}_{\perp}\right.$, and FA). The impact of LPS exposure on diffusion, before the onset of myelination, produced diffuse alteration of the developing CC and PVWM. It is important to stress that the diffusion data were acquired on ex vivo fixed brains, and as such the diffusivity values are lower than those observed in vivo, whereas FA values remain unchanged (31). The reductions in FA observed following LPS were largely due to an increase of $\mathrm{D}_{\wedge}$ (i.e., increased water mobility perpendicular to the mean direction of diffusion) similar to in vivo data after LPS exposure in rat pups (18) and MR studies in preterm infants with WM injury $(17,32,33)$. Increased $\mathrm{D}_{\wedge}$ was attributed to a loss of myelin in adult animal models (34). Nevertheless, WM myelination has just started at the developmental stage used in this study (11). Therefore, 


\section{Articles | van de Looij et al.}

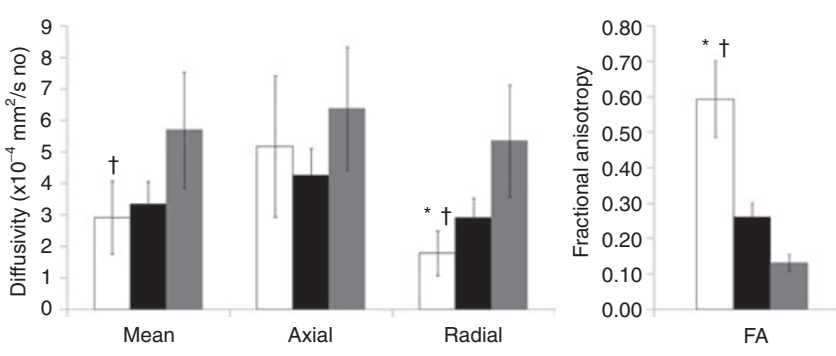

Figure 7. Mean (MD), axial $\left(D_{\mu}\right)$, and radial $\left(D_{\perp}\right)$ diffusivity as well as fractional anisotropy (FA) \pm SD measured in the necrotic lesion (black) and cyst (gray) of lipopolysaccharide-exposed sheep brains as well as in the Sham counterpart (white). Anisotropy was significantly reduced in the both lesion types due to predominant radial diffusivity increase. Only for the cyst was a significant mean diffusivity reduction observed $\left({ }^{*} P<0.05\right.$, Mann-Whitney test, Sham vs. necrotic lesion; ${ }^{\dagger} P<0.05$, Mann-Whitney test, Sham vs. cyst).

changes in $\mathrm{D}_{\wedge}$ are likely to represent a more global WM structure disruption, with increased interaxonal space and altered axonal ensheathment due to the loss of oligodendrocytes (11), leading to facilitated $\mathrm{D}_{\wedge}$ rather than altered myelination. Of note, all the DTI-derived parameters in the CC were found to be correlated with our global WM injury score, suggesting that the degree of microstructural changes in a localized WM tract relates to more widespread damage and its severity.

We observed three types of WM lesions characterized by diffuse and focal MRI characteristics. These lesions closely correspond to the WM lesions observed in PVL of human brains. For focal lesions, it is important to note that regions of interest were manually delineated through the overt lesions, and careful attention was paid to avoid adjacent tissue.

\section{Diffuse Changes}

Diffuse $\mathrm{T}_{2}$ hyperintensities and $\mathrm{T}_{1}$ hypointensities were present in the subcortical WM (Figure 5). These signal changes had microstructural correlates of increased $\mathrm{D}_{\perp}$ and decreased FA. These lesions show the same properties as the diffuse WM hyperintensity well described in human premature infants (35). Histological correlates showed an alteration of tissue structure as demonstrated by reduced $\mathrm{AF} / \mathrm{T}$ and neurofilament stain and organization. Only minor APP staining was observed, suggesting that remaining axons in these areas are not altered and do not result in accumulation of axonally transported APP proteins. Furthermore, previous findings showed an overall decrease in number of oligodendrocytes in the PVWM following LPS exposure, with diffuse altered myelination (11). Similar diffuse WM injuries characterized by $\mathrm{T}_{2}$ hyperintensities were shown in the fetal sheep after global ischemia $(11,19)$.

\section{Focal Necrosis}

Focal $T_{2}$ hypointensities and $T_{1}$ hyperintensities indicative of necrotic lesions were observed in the PVWM (Figures 6 and 7 -necrotic lesion). These signal changes may arise from high lipid content in macrophages and activated microglia (11) with lipids, or from a higher cellular density in the lesion as compared with the surrounding tissue as seen on the AF/T-stained tissue. Furthermore, in these regions, FA was reduced (closed to the value observed in $\mathrm{GM}$ ) and $\mathrm{D}_{\perp}$ was increased, corresponding to neuropathological modifications seen in these areas. Histologically, these focal lesions were characterized by reduced glial fibrillary acidic protein and antiphos-neurofilament staining, suggesting that both astrocytes and axons are damaged. In support of axonal damage, focal necrotic lesions exhibited a marked accumulation of APP, with minor alteration of the pattern of fiber tracts around the lesion. A similar pattern of focal WM lesions defined by hyposignal $\mathrm{T}_{2}$, increased $\mathrm{MD}$, and decreased FA was observed in the preterm fetal sheep following global ischemia (19), and these lesions correspond to areas of activated microglia (11). Following either LPS or hypoxia-ischemia challenge, these focal lesions exhibit a similar location and anatomy within the PVWM to those of the punctate lesions observed in human premature infants $(36,37)$, and include a similar reduction in FA (5). To the best of our knowledge, this is the first evidence of WM lesions similar to the punctate lesions in humans in an animal model of preterm inflammatory brain injury.

\section{Cystic Lesions}

Focal $T_{1}$ hypointensities and $T_{2}$ hyperintensities in the WM (Figures 6 and 7-cystic lesion) exhibited an overall increase in all diffusivity values with a dramatic FA reduction. WM cysts were reported to appear as acute changes in $\mathrm{T}_{1}, \mathrm{~T}_{2}$, and DTIderived parameters (38). In vivo, the content of unhindered, isotropically diffusing cerebrospinal fluid in these cavities translates as a marked increase in $\mathrm{MD}$, as well as strong $\mathrm{T}_{1}$ hypointensities and $\mathrm{T}_{2}$ hyperintensities comparable with the cerebrospinal fluid in ventricles. Obvious disruption of fiber tract organization around the lesions was seen on eigenvector maps. Periventricular cysts and similar disruption of WM tracts are a hallmark of cystic PVL seen in preterm infants leading to significant motor visual and cognitive impairment $(3,16,39)$.

\section{Conclusion}

This work provides an advanced MRI-based delineation of the brain changes following experimental LPS exposure in an immature gyrified brain. Different types of brain damage were observed by MRI and were characterized on anatomical, microstructural, and histopathological levels. The pattern of lesions observed in LPS-treated fetal sheep mimics the pattern of injuries seen in premature infants very well, confirming the relevance of such a model. WM lesions were extended throughout the brain with diffuse and focal components. Reduced FA and increased radial diffusivity $\left(D_{\perp}\right)$ values revealed widespread alterations in developing WM after fetal exposure to LPS before the onset of myelination. More specifically, we show the ability of DTI to classify different types of brain lesions following LPS exposure with specific changes of DTI-derived parameters as well as of the eigenvectors tracking between cysts and necrotic lesions.

\section{MATERIALS AND METHODS}

\section{Animal Preparation}

A total of 18 fetal sheep were operated on at 97-99 d of gestation (corresponding to human preterm at $28-32 \mathrm{wk}$ of gestation; term $=$ $147 \mathrm{~d})$ under general anesthesia $\left(1.5 \%\right.$ isoflurane in $\left.\mathrm{O}_{2}\right)$ using sterile 
techniques (12). Fetuses were randomly assigned to receive i.v. bolus infusion of either saline vehicle (Sham group; $n=9$ ) or $200 \mathrm{ng} / \mathrm{kg}$ Escherichia coli LPS (055:B4; Sigma-Aldrich, St. Louis, MO; LPS group; $n=9$ ). At $10 \mathrm{~d}$ after LPS, the ewe and fetus were killed by i.v. overdose of sodium pentobarbitone. Fetal brains were perfusion-fixed in situ with $0.9 \% \mathrm{NaCl}$ solution and then $4 \% \mathrm{PFA}$ in $0.1 \mathrm{~mol} / \mathrm{l}$ phosphate buffer (Histofix; Histolab, Gothenburg, Sweden). Brains were postfixed in 4\% PFA for 6-9 mo before MRI. All experimentation was approved by the Ethical Animal Committee of Gothenburg, Sweden, (no. 307-2006) and conformed to international guidelines on the ethical use of animals.

\section{MR Experiments}

For all MR experiments, the brains were soaked with fomblin (Fomblin Profludropolyether; Ausimont, Thorofare, NJ) during scanning. $\mathrm{T}_{1}$ and $\mathrm{T}_{2}$-weighted images were acquired on a 3T Siemens Trio System (Siemens, Erlangen, Germany) with a standard wrist coil. $\mathrm{T}_{1} \mathrm{~W}$ images were acquired with magnetization prepared rapid gradient echo sequence (inversion time equal to $600 \mathrm{~ms}$, TE $=3.17 \mathrm{~ms}$, and flip angle $8^{\circ}$ ) and $\mathrm{T}_{2} \mathrm{~W}$ images were acquired with a turbo spin echo sequence $\left(\mathrm{TR}=4,910 \mathrm{~ms}\right.$, TE $=141 \mathrm{~ms}$, flip angle $=150^{\circ}$, echo train length $=15$ ). The resolution was $0.4 \times 0.4 \times 0.6 \mathrm{~mm}^{3}$ for $\mathrm{T}_{1} \mathrm{~W}$ and $\mathrm{T}_{2} \mathrm{~W}$ images for an acquisition time of $7 \mathrm{~min} 20 \mathrm{~s}$ and $9 \mathrm{~min}$, respectively.

DTI experiments were performed on an actively shielded 9.4T/31-cm magnet (Varian, Palo Alto, CA/Magnex Scientific, Oxford, UK) equipped with $12-\mathrm{cm}$ gradient coils $(400 \mathrm{mT} / \mathrm{m}, 120 \mu \mathrm{s})$ with a custom-built solenoid 50 -mm radio frequency coil. Diffusion gradients were applied along six spatial directions with a $b$ value set to $1,971 \mathrm{~s} / \mathrm{mm}^{2}$. Scans were averaged two times with TE/TR $=35 / 11,000 \mathrm{~ms}$ for an acquisition time of around $12 \mathrm{~h}$. The resolution was $0.19 \times 0.19 \times 1 \mathrm{~mm}^{3}$.

\section{Volume Segmentation}

Quantitative measurements of cerebral tissue volumes (cortical GM, basal ganglia, unmyelinated WM, and nonliving liquid) were performed using a nonparametric signal intensity estimator with $\mathrm{k}$-nearest neighbor classification, based both on the MRI signal intensity of the registered $\mathrm{T}_{2} \mathrm{~W}$ and $\mathrm{T}_{1} \mathrm{~W}$ images, and on anatomic location to differentiate cortex and central GM according to basal ganglia atlases $(13,22)$. Initial estimates for the tissue classifiers were manually defined.

\section{DTI Analysis}

Customised Matlab scripts (The Mathworks, Natick, MA) were written to derive the $\mathrm{D}_{\perp}, \mathrm{D}_{\| \mid}, \mathrm{MD}$, and FA from the diffusion tensor. To visualize the orientation of the fiber bundles, direction-encoded color maps were generated based on the principal eigenvector direction. Free software, MedInria DTI track (http://www-sop.inria.fr/asclepios/software/MedINRIA/), was used for the computation and the display of the principal eigenvectors (i.e., the vector corresponding to the principal diffusion direction).

Six regions of interest were manually delineated on directionencoded color maps: the CC, the PVWM, the internal capsule, the external capsule, and the cortex at the anterior, median, and posterior levels of the brain. (See Figure 2 for the corresponding image-planes of the different brain levels.) The corpus callosum thickness was manually measured with ImageJ (http://rsbweb.nih.gov/ij/) on directionencoded color maps at the posterior level of the brain (posterior level on Figure 2).

For each animal, $\mathrm{T}_{1} \mathrm{~W}$ and $\mathrm{T}_{2} \mathrm{~W}$ images were carefully observed by three different clinicians (G.A.L., P.S.H., and S.V.S.) to assess the presence of signal abnormalities corresponding to WM damage. For each animal, a region of interest accurately encompassing the lesion was manually delineated on the corresponding $\mathrm{T}_{2} \mathrm{~W}$ image. The different patterns recognized were hyper $\mathrm{T}_{2}$ and hypo $\mathrm{T}_{1}$ (focal and diffuse lesions), and hypo $\mathrm{T}_{2}$ and hyper $\mathrm{T}_{1}$ (focal lesions only). DTI-derived parameters measured in these lesions were compared with frontoparietal PVWM and subcortical WM values measured in the Sham group for focal and diffuse lesions, respectively.

A global WM abnormality score for each sheep, including diffuse WM signal abnormality, PVWM volume loss, cystic lesions, or gliotic lesions, was used (11) for correlations between individual injury score and DTI-derived parameters (FA, MD, $\mathrm{D}_{\downarrow}$, and $\mathrm{D}_{\|}$), corpus callosum thickness, and WM volumes using Matlab.

\section{Histopathology}

Histology was performed 3-6 mo after the MRI scans. For each animal, a series of evenly spaced paraffin embedded coronal sections $(10 \mu \mathrm{m}$ thick, section interval $=150)$ were collected from the level of the anterior caudoputamen to the posterior aspects of the hippocampus of the fetal brain. Sections were stained with AF/T for general tissue morphological (12) and immunohistochemical analysis to detect astrocytes (anti-mouse glial fibrillary acidic protein, 1:250; SigmaAldrich), neurofilament (monoclonal antiphos-neurofilaments, SMI312, 1:2000; Covance, Emeryville, CA), and APP (mouse anti-Alzheimer precursor protein A4, 1:100; Millipore, Billerica, MA). Immunohistochemical staining protocols were previously described (11). In brief, following antigen retrieval primary antibodies were incubated for $1 \mathrm{~h}$ at room temperature and visualized using appropriate biotinylated secondary antibodies, Vectorstain $\mathrm{ABC}$ system (Vector Laboratories, Burlingame, CA) and 3-3-diaminobenzidine with nickel enhancement. Areas with changes on MRI were identified in histological sections. The sections were analyzed under an Olympus BX60 microscope equipped with an Olympus DP50 cooled digital camera, and images were captured using Micro Image version 4.0 (Olympus Optical, Tokyo, Japan).

\section{Statistical Analysis}

Differences in DTI-derived parameters (diffusivity values and FA) and tissue volumes, as well as focal and diffuse lesions detected by abnormal $\mathrm{T}_{1}$ and $\mathrm{T}_{2}$ signals between the two groups, were assessed by a Mann-Whitney test. Results are presented as mean \pm SD. All statistical significances were set as $P<0.05$, except for DTI-derived parameters where a Bonferroni correction (i.e., correction taking into account the measures from multiple planes in the same animal) was applied, leading to a significance level of 0.017 .

\section{STATEMENT OF FINANCIAL SUPPORT}

This work was supported by Swiss National Fund grant 31003A-112233 and Swiss National Science Foundation (SPUM) grant 33CM30-124101; the European Association Against Leukodystrophies (ELA) Foundation, Nancy, France; the Centre d'Imagerie Biomédicale of the Université de Lausanne (UNIL), Université de Genève (UNIGE), Hôpitaux Universitaires de Genève (HUG), Centre Hospitalier Universitaire Vaudois (CHUV), and Ecole Polytechnique Fédérale de Lausanne (EPFL); the Leenards and Jeantet Foundations; the NEOBRAIN Consortium under the Sixth Framework Programme of the European Commission (grant 2006-036534); the Swedish Research Council (grant 2006-2783); the Free Mason Foundation; the Åhlén's Foundation; and the Wilhelm and Martina Lundgren Foundation.

\section{REFERENCES}

1. Banker BQ, Larroche JC. Periventricular leukomalacia of infancy. A form of neonatal anoxic encephalopathy. Arch Neurol 1962;7:386-410.

2. Maalouf EF, Duggan PJ, Counsell SJ, et al. Comparison of findings on cranial ultrasound and magnetic resonance imaging in preterm infants. Pediatrics 2001;107:719-27.

3. Volpe JJ. Brain injury in premature infants: a complex amalgam of destructive and developmental disturbances. Lancet Neurol 2009;8:110-24.

4. Schmidt R, Fazekas F, Kapeller P, Schmidt H, Hartung HP. MRI white matter hyperintensities: three-year follow-up of the Austrian Stroke Prevention Study. Neurology 1999;53:132-9.

5. Bassi L, Chew A, Merchant N, et al. Diffusion tensor imaging in preterm infants with punctate white matter lesions. Pediatr Res 2011;69:561-6.

6. Counsell SJ, Allsop JM, Harrison MC, et al. Diffusion-weighted imaging of the brain in preterm infants with focal and diffuse white matter abnormality. Pediatrics 2003;112(1 Pt 1):1-7.

7. Dammann O, Phillips TM, Allred EN, et al.; ELGAN STUDY INVESTIGATORS. Mediators of fetal inflammation in extremely low gestational age newborns. Cytokine 2001;13:234-9.

8. Hagberg H, Mallard C, Jacobsson B. Role of cytokines in preterm labour and brain injury. BJOG 2005;112:Suppl 1:16-8. 
9. Cai Z, Pan ZL, Pang Y, Evans OB, Rhodes PG. Cytokine induction in fetal rat brains and brain injury in neonatal rats after maternal lipopolysaccharide administration. Pediatr Res 2000;47:64-72.

10. Debillon T, Gras-Leguen C, Vérielle V, et al. Intrauterine infection induces programmed cell death in rabbit periventricular white matter. Pediatr Res 2000;47:736-42.

11. Dean JM, van de Looij Y, Sizonenko SV, et al. Delayed cortical impairment following lipopolysaccharide exposure in preterm fetal sheep. Ann Neurol 2011;70:846-56.

12. Mallard C, Welin AK, Peebles D, Hagberg H, Kjellmer I. White matter injury following systemic endotoxemia or asphyxia in the fetal sheep. Neurochem Res 2003;28:215-23.

13. Hüppi PS, Warfield S, Kikinis R, et al. Quantitative magnetic resonance imaging of brain development in premature and mature newborns. Ann Neurol 1998;43:224-35.

14. Inder TE, Huppi PS, Warfield S, et al. Periventricular white matter injury in the premature infant is followed by reduced cerebral cortical gray matter volume at term. Ann Neurol 1999;46:755-60.

15. Inder TE, Warfield SK, Wang H, Hüppi PS, Volpe JJ. Abnormal cerebral structure is present at term in premature infants. Pediatrics 2005;115:286-94.

16. Ment LR, Hirtz D, Hüppi PS. Imaging biomarkers of outcome in the developing preterm brain. Lancet Neurol 2009;8:1042-55.

17. Hüppi PS, Murphy B, Maier SE, et al. Microstructural brain development after perinatal cerebral white matter injury assessed by diffusion tensor magnetic resonance imaging. Pediatrics 2001;107: 455-60.

18. Lodygensky GA, West T, Stump M, Holtzman DM, Inder TE, Neil JJ. In vivo MRI analysis of an inflammatory injury in the developing brain. Brain Behav Immun 2010;24:759-67.

19. Riddle A, Dean J, Buser JR, et al. Histopathological correlates of magnetic resonance imaging-defined chronic perinatal white matter injury. Ann Neurol 2011;70:493-507.

20. Sizonenko SV, Camm EJ, Garbow JR, et al. Developmental changes and injury induced disruption of the radial organization of the cortex in the immature rat brain revealed by in vivo diffusion tensor MRI. Cereb Cortex 2007;17:2609-17.

21. van de Looij Y, Mauconduit F, Beaumont M, et al. Diffusion tensor imaging of diffuse axonal injury in a rat brain trauma model. NMR Biomed 2012;25:93-103.

22. Warfield SK, Kaus M, Jolesz FA, Kikinis R. Adaptive, template moderated, spatially varying statistical classification. Med Image Anal 2000;4:43-55.

23. Nosarti C, Mechelli A, Herrera A, et al. Structural covariance in the cortex of very preterm adolescents: a voxel-based morphometry study. Hum Brain Mapp 2011;32:1615-25.
24. Zubiaurre-Elorza L, Soria-Pastor S, Junque C, et al. Gray matter volume decrements in preterm children with periventricular leukomalacia. Pediatr Res 2011;69:554-60.

25. Thompson DK, Inder TE, Faggian N, et al. Characterization of the corpus callosum in very preterm and full-term infants utilizing MRI. Neuroimage 2011;55:479-90.

26. Anderson NG, Laurent I, Woodward LJ, Inder TE. Detection of impaired growth of the corpus callosum in premature infants. Pediatrics 2006;118:951-60.

27. Woodward LJ, Anderson PJ, Austin NC, Howard K, Inder TE. Neonatal MRI to predict neurodevelopmental outcomes in preterm infants. N Engl J Med 2006;355:685-94.

28. Caldú X, Narberhaus A, Junqué C, et al. Corpus callosum size and neuropsychologic impairment in adolescents who were born preterm. J Child Neurol 2006;21:406-10

29. Ajayi-Obe M, Saeed N, Cowan FM, Rutherford MA, Edwards AD. Reduced development of cerebral cortex in extremely preterm infants. Lancet 2000;356:1162-3.

30. Brown NC, Inder TE, Bear MJ, Hunt RW, Anderson PJ, Doyle LW. Neurobehavior at term and white and gray matter abnormalities in very preterm infants. J Pediatr 2009;155:32-8, 38.e1.

31. Sun SW, Neil JJ, Song SK. Relative indices of water diffusion anisotropy are equivalent in live and formalin-fixed mouse brains. Magn Reson Med 2003;50:743-8.

32. Cheong JL, Thompson DK, Wang HX, et al. Abnormal white matter signal on MR imaging is related to abnormal tissue microstructure. AJNR Am J Neuroradiol 2009;30:623-8.

33. Counsell SJ, Shen Y, Boardman JP, et al. Axial and radial diffusivity in preterm infants who have diffuse white matter changes on magnetic resonance imaging at term-equivalent age. Pediatrics 2006;117:376-86.

34. Song SK, Sun SW, Ramsbottom MJ, Chang C, Russell J, Cross AH. Dysmyelination revealed through MRI as increased radial (but unchanged axial) diffusion of water. Neuroimage 2002;17:1429-36.

35. Maalouf EF, Duggan PJ, Rutherford MA, et al. Magnetic resonance imaging of the brain in a cohort of extremely preterm infants. J Pediatr 1999;135:351-7.

36. Cornette LG, Tanner SF, Ramenghi LA, et al. Magnetic resonance imaging of the infant brain: anatomical characteristics and clinical significance of punctate lesions. Arch Dis Child Fetal Neonatal Ed 2002;86:F171-7.

37. Rutherford MA, Supramaniam V, Ederies A, et al. Magnetic resonance imaging of white matter diseases of prematurity. Neuroradiology 2010;52:505-21

38. Pierpaoli C, Barnett A, Pajevic S, et al. Water diffusion changes in Wallerian degeneration and their dependence on white matter architecture. Neuroimage 2001;13(6 Pt 1):1174-85.

39. Ramenghi LA, Rutherford M, Fumagalli M, et al. Neonatal neuroimaging: going beyond the pictures. Early Hum Dev 2009;85(10 Suppl):S75-7. 